Regards sur l'économie allemande

Bulletin économique du CIRAC

$118-119 \mid 2015$

Varia

\title{
Élections au Bundestag : droit de vote à 16 ans ?
}

\section{Isabelle Bourgeois}

\section{OpenEdition}

Journals

Édition électronique

URL : http://journals.openedition.org/rea/4902

DOI : $10.4000 /$ rea.4902

ISSN : 1965-0787

\section{Éditeur}

CIRAC

\section{Édition imprimée}

Date de publication : 31 décembre 2015

Pagination : 40-41

ISSN : 1156-8992

\section{Référence électronique}

Isabelle Bourgeois, «Élections au Bundestag : droit de vote à 16 ans? 》, Regards sur l'économie allemande [En ligne], 118-119 | décembre 2015, mis en ligne le 31 décembre 2017, consulté le 15 septembre 2020. URL : http://journals.openedition.org/rea/4902

Ce document a été généré automatiquement le 15 septembre 2020.

(c) CIRAC 


\title{
Élections au Bundestag : droit de vote à 16 ans?
}

\author{
Isabelle Bourgeois
}

1 Depuis de nombreuses années, on se pose en Allemagne la question de savoir s'il convient d'abaisser l'âge du droit de vote en réponse à un taux d'abstention élevé chez les primo-électeurs, et plus généralement les plus jeunes, qui risque de les détourner durablement de la participation aux élections lorsqu'ils seront plus âgés, un enjeu clé pour l'avenir de la démocratie dans une société vieillissante. Et par-delà, pour la cohésion de la société et l'adhésion des citoyens aux institutions, car le taux d'abstention est particulièrement marqué chez les jeunes des milieux défavorisés. Le droit de vote à 16 ans permettrait ainsi de remédier à cette forme d'(auto)exclusion. A titre d'exemple, lors des dernières élections au Bundestag (2013), les tranches d'âge inférieures à 40 ans étaient particulièrement sous-représentées parmi les votants. De ce fait notamment, le taux de participation n'avait atteint que $72 \%$.

\section{Comment accroître le taux de participation des moins de 40 ans?}

2 Comment donc accroître à l'avenir le taux de participation des moins de 40 ans ? En abaissant dès maintenant l'âge du droit de vote à 16 ans, préconise une étude réalisée par la Fondation Bertelsmann en coopération avec l'Institut für Demoskopie d'Allensbach, qui a réalisé les sondages ("Wählen ab 16 »). Le taux de participation aux élections du Bundestag pourrait de la sorte être porté à $80 \%$ en 2046. La demande des 16-17 ans est forte en effet: alors qu'ils se détournaient encore largement de la vie politique en 2002 (seuls $30 \%$ d'entre eux se disaient intéressés/ très intéressés), ils sont aujourd'hui 41 \% à s'y intéresser. Mais cet intérêt dépend du niveau d'études : plus il est élevé, plus l'envie de participer est forte. Cela plaide donc pour un accompagnement suivi, notamment au sein du système scolaire qui devrait développer encore plus l'instruction civique. 
3 L'étude s'inspire de plusieurs cas où le droit de vote à 16 ans existe déjà. Ainsi, en Autriche, il a été introduit en 2008, pour tous les scrutins sauf les élections européennes. En Allemagne, dans la plupart des Länder, les jeunes de 16 ans participent aux élections municipales - depuis 1996 en Basse-Saxe, le pionnier en la matière. Dans trois Länder, ils participent également aux élections parlementaires régionales : dans les villes-Etat de Brême et Hambourg depuis respectivement 2011 et 2015, ainsi que dans le Brandebourg, depuis 2014. Mais pour voter aux élections du Bundestag, il faut toujours avoir 18 ans.

\section{Droit de vote à 16 ans ? Les Allemands sont réticents}

4 Mais qu'en pensent les Allemands? Si les 16-17 ans sont à l'évidence d'ardents défenseurs de l'abaissement de cet âge d'entrée dans la vie du citoyen adulte, il n'en va pas de même des autres : un Allemand sur huit s'y oppose, le refus croissant avec l'âge. L'appartenance politique joue un rôle également: alors que les partisans de l'Union CDU/CSU se montrent réticents, cette idée séduit un peu plus les Verts, l'AfD et Die Linke. Par religion : les protestants y sont plus ouverts que les catholiques ou les sansconfession.

Quant au rôle du collège/lycée dans la préparation des échéances électorales, il suscite des réponses favorables. Sans surprise, les 16-17 ans sont de loin les plus désireux d'être informés des enjeux du scrutin et de débattre des programmes électoraux en classe.

Le thème des élections devrait-il être traité au collège/au lycée ?

Voici deux personnes qui discutent de la question de savoir si les questions politiques devraient jouer un plus grand rôle dans l'enseignement. Laquelle des deux dit plutôt ce que vous pensez vous aussi ? Celle du haut ou celle du bas? Personne du haut: Je trouve qu'il est important qu'en classe, on ne se contente pas d'enseigner aux élèves le système politique, mais qu'on les informe aussi, à l'approche d'une élection, sur les enjeux du scrutin et sur les programmes des différents partis. De cette manière, on peut éveiller l'intérêt des jeunes pour la vie politique.

Personne du bas : Je vois les choses autrement. Bien sûr, il est important que les grands principes d'organisation de notre système politique soient abordés en classe. Mais le collège/lycée n'est pas le lieu pour aller au-delà et discuter par exemple des enjeux d'élections à venir ou des programmes des partis en présence.

\begin{tabular}{|l|c|c|c|c|c|c|c|c|c|c|}
\hline & RFA & Ouest & Est & Hommes & Femmes & $16-17$ a. & $\begin{array}{c}16-29 \\
\text { a. }\end{array}$ & $30-44$ a. & $45-59$ a. & 60 a. et + \\
\hline $\begin{array}{l}\text { P. du } \\
\text { haut }\end{array}$ & $\mathbf{6 1 , 5} \%$ & $\mathbf{6 1 , 5} \%$ & $\mathbf{6 1 , 5} \%$ & $\mathbf{6 0 , 7} \%$ & $\mathbf{6 2 , 1} \%$ & $\mathbf{8 1 , 0} \%$ & $\mathbf{6 8 , 2} \%$ & $\mathbf{5 7 , 1} \%$ & $\mathbf{6 2 , 5} \%$ & $\mathbf{5 9 , 7} \%$ \\
\hline $\begin{array}{l}\text { P. du } \\
\text { bas }\end{array}$ & $\mathbf{2 6 , 8} \%$ & $\mathbf{2 7 , 3} \%$ & $\mathbf{2 4 , 8} \%$ & $\mathbf{2 7 , 2} \%$ & $\mathbf{2 6 , 5} \%$ & $\mathbf{5 , 7} \%$ & $\mathbf{1 9 , 4} \%$ & $\mathbf{3 0 , 4} \%$ & $\mathbf{2 8 , 9} \%$ & $\mathbf{2 8 , 9} \%$ \\
\hline $\begin{array}{l}\text { Indécis, } \\
\text { nsp }\end{array}$ & $11,7 \%$ & $11,2 \%$ & $13,7 \%$ & $12,0 \%$ & $11,4 \%$ & $13,3 \%$ & $12,4 \%$ & $12,5 \%$ & $11,1 \%$ & $11,4 \%$ \\
\hline & $100 \%$ & $100 \%$ & $100 \%$ & $100 \%$ & $100 \%$ & $100 \%$ & $100 \%$ & $100 \%$ & $100 \%$ & $100 \%$ \\
\hline
\end{tabular}




\begin{tabular}{|c|c|c|c|c|c|c|c|c|c|c|}
\hline & $\begin{array}{l}\text { Ouvrier } \\
\text { spécial. }\end{array}$ & $\begin{array}{l}\text { Ouvrier } \\
\text { qualifié }\end{array}$ & $\begin{array}{l}\text { Employé, } \\
\text { fonctionn. }\end{array}$ & Cadre & Indépend. & $\begin{array}{l}\text { D'origine } \\
\text { immigrée }\end{array}$ & $\begin{array}{c}\text { De } \\
\text { souche }\end{array}$ & Catholique & Protestant & $\begin{array}{c}\text { Autre/ } \\
\text { sans } \\
\text { confession }\end{array}$ \\
\hline $\begin{array}{l}\text { P. du } \\
\text { haut }\end{array}$ & $52,8 \%$ & $54,8 \%$ & $66,2 \%$ & $59,6 \%$ & $58,6 \%$ & $56,4 \%$ & $61,8 \%$ & $61,4 \%$ & $61,8 \%$ & $61,3 \%$ \\
\hline $\begin{array}{l}\text { P. du } \\
\text { bas }\end{array}$ & $24,4 \%$ & $29,8 \%$ & $25,4 \%$ & $28,2 \%$ & $28,6 \%$ & $28,8 \%$ & $26,8 \%$ & $26,9 \%$ & $25,9 \%$ & $27,6 \%$ \\
\hline \multirow[t]{3}{*}{$\begin{array}{l}\text { Indécis, } \\
\text { nsp }\end{array}$} & $22,8 \%$ & $15,3 \%$ & $8,4 \%$ & $12,2 \%$ & $12,8 \%$ & $14,8 \%$ & $11,4 \%$ & $11,7 \%$ & $12,3 \%$ & $11,1 \%$ \\
\hline & $100 \%$ & $100 \%$ & $100 \%$ & $100 \%$ & $100 \%$ & $100 \%$ & $100 \%$ & $100 \%$ & $100 \%$ & $100 \%$ \\
\hline & $\begin{array}{c}\mathrm{CDU} / \\
\mathrm{CSU}\end{array}$ & SPD & FDP & $\begin{array}{l}\text { B. 90/ } \\
\text { Die } \\
\text { Grünen }\end{array}$ & Die Linke & AfD & & $\begin{array}{l}\text { S'intéresse } \\
\text { à vie polit. }\end{array}$ & Désintérêt & \\
\hline $\begin{array}{l}\text { P. du } \\
\text { haut }\end{array}$ & $61,2 \%$ & $63,6 \%$ & $69,7 \%$ & $67,4 \%$ & $56,4 \%$ & $58,5 \%$ & & $68,1 \%$ & $54,9 \%$ & \\
\hline $\begin{array}{l}\text { P. du } \\
\text { bas }\end{array}$ & $28,1 \%$ & $23,6 \%$ & $12,7 \%$ & $22,0 \%$ & $34,6 \%$ & $28,2 \%$ & & $25,5 \%$ & $28,1 \%$ & \\
\hline \multirow{2}{*}{$\begin{array}{l}\text { Indécis, } \\
\text { nsp }\end{array}$} & $10,7 \%$ & $12,8 \%$ & $17,6 \%$ & $10,6 \%$ & $8,9 \%$ & $13,3 \%$ & & $6,4 \%$ & $16,9 \%$ & \\
\hline & $100 \%$ & $100 \%$ & $100 \%$ & $100 \%$ & $100 \%$ & $100 \%$ & & $100 \%$ & $100 \%$ & \\
\hline
\end{tabular}

NB : sondage représentatif effectué du $7^{\text {er }}$ au 12 septembre 2015 auprès d'un échantillon représentatif de 1402 personnes germanophones de 16 ans et plus.

Source des données : Institut für Demoskopie d'Allensbach/ Bertelsmann-Stiftung, rapport Wählen mit 16 (www.bertelsmann-stiftung.de/fileadmin/files/BSt/Publikationen/GrauePublikationen/

ZD_Studie_Waehlen_ab_16_2015.pdf).

\section{Multiplier les « élections-junior »}

6 Mais le collège/lycée n'est qu'un lieu parmi d'autres de la formation de la volonté politique des jeunes citoyens. En Allemagne aussi, le lieu privilégié des débats comme de la socialisation politique reste la famille, suivie par le cercle des amis. Or, pour accroitre la participation des jeunes, il s'agit de mener une politique de longue haleine afin de évelopper leurs « compétences politiques» (Petersen/Bertelsmann-Stiftung).

7 La multiplication d'actions d'éducation civique à l'instar de ces «élections-junior " (Juniorwahlen) qui s'adressent aux 15-16 ans dans leur environnement scolaire devrait accroitre leur motivation si elles sont menées plus systématiquement encore. Introduites en 1999, et pratiquées dans toute l'Allemagne, elles sont programmées dans les semaines précédant les élections parlementaires régionales, fédérales et les élections européennes et débouchent, après de nombreux débats, sur un scrutin. Quelque 1,8 million de jeunes a ainsi pu acquérir une "expérience pratique de la 
démocratie». Voilà comment l'Allemagne affronte le défi du vieillissement démographique sous l'angle de la démocratie aussi.

INDEX

Mots-clés : Bundestag, élection, droit de vote 